\title{
Frequency of chondrocalcinosis of the knees in asymptomatic hyperuricaemia and rheumatoid arthritis: a controlled study
}

\author{
P. HOLLINGWORTH, ${ }^{1}$ P. L. WILLIAMS,$^{2}$ AND J. T. SCOTT \\ From the ${ }^{1}$ Charing Cross Hospital and Kennedy Institute of Rheumatology, London W6, and ${ }^{2}$ Northwick Park \\ Hospital, Harrow, Middlesex
}

SUMMARY An association has been reported between urate gout and chondrocalcinosis. Chondrocalcinosis was detected in knee radiographs of 8 of 138 gouty patients compared with none of 142 age matched nongouty normouricaemic controls $(\mathrm{p}<0.025)$. To define this association further, knee radiographs were examined of 84 patients with asymptomatic hyperuricaemia who had been carefully age matched with the gouty and control groups. Chondrocalcinosis of the knees was detected in only one patient, the difference from the control group not being significant. It therefore appears that there is an association of chondrocalcinosis with gouty arthritis, but not with asymptomatic hyperuricaemia. An association between chondrocalcinosis and rheumatoid arthritis has been suggested, but the studies were not controlled for age. To re-examine this implied association, knee radiographs were inspected of 127 patients with rheumatoid arthritis who had been age matched with the control group. Chondrocalcinosis of the knees was detected in only one patient, a difference from the control group which is not statistically significant.

The frequency of chondrocalcinosis has been conclusively shown to be increased in patients with urate gout in a companion study, ${ }^{1}$ but unlike previous similar studies ${ }^{2}{ }^{3}$ this was carefully controlled for age. The mechanism of this association has been postulated to be either a predisposition to the local deposition of pyrophosphate due to the presence of urate crystals, perhaps acting as a nucleating agent ${ }^{4}$ or factors which predispose to the deposition of both kinds of crystals. ${ }^{5} 7$

It is also possible that hyperuricaemia in itself predisposes to chondrocalcinosis as well as to gout. In order to examine this possibility a radiological survey was undertaken of the knees of patients with asymptomatic hyperuricaemia who had been age matched with the gouty group of our companion study, and the frequencies of chondrocalcinosis of the 2 groups were compared.

The frequency of chondrocalcinosis has also been reported to be increased in patients with rheumatoid arthritis, ${ }^{28}$ though neither of these studies was controlled for age and their conclusions may be invalid. This implied association was re-examined by comparing the frequency of chondrocalcinosis of knee radiographs of the control group with that of an agematched group of patients with rheumatoid arthritis.

Accepted for publication $10 \mathrm{July} 1981$.

Correspondence to Dr J. T. Scott, Kennedy Institute of Rheumatology, Bute Gardens, London W6 7DW.

\section{Patients and methods}

Patients with hyperuricaemia were identified by a computer print-out which included every reading of hyperuricarmia greater than $420 \mu \mathrm{mol} / 1$ recorded in Northwick Park Hospital between 1973 and 1979. Medical records were examined and patients were excluded who had a history of gout or an identifiable cause of transient hyperuricaemia such as diuretic therapy, dieting, or renal failure. Patients were further questioned to exclude a history of gout when they attended for $x$-ray. The diagnoses of these patients at the time of estimation of serum uric acid are given in Table 1 .

Patients with rheumatoid arthritis were identified by a computer print-out which included every patient who had been admitted to Northwick Park Hospital with the disease. Medical records were examined and patients were selected who had definite or classical

Table 1 The diagnoses of patients with asymptomatic hyperuricaemia

\begin{tabular}{lr}
\hline Minor surgical and orthopaedic & 28 \\
Hyperlipidaemia & 13 \\
Diseases caused by atheroma & 8 \\
Endocrine (not hypothyroid) & 5 \\
Renal colic (not urate stones or hyperparathyroidism) & 3 \\
Obesity (not dieting) & 3 \\
Others & 24 \\
\hline
\end{tabular}


Table 2 Details of patients with gout, asymptomatic hyperuricaemia, and the control group

\begin{tabular}{|c|c|c|c|}
\hline & Gout & $\begin{array}{l}\text { Asymptomatic } \\
\text { hyperuricaemia }\end{array}$ & Control group \\
\hline Number & 138 & 84 & 142 \\
\hline Male & 136 & 71 & 142 \\
\hline Female & 2 & 13 & 0 \\
\hline Age (years) ${ }^{2}$ & $55 \cdot 2 \pm 12 \cdot 9$ & $54 \cdot 5 \pm 14 \cdot 3$ & $54 \cdot 9 \pm 13 \cdot 4$ \\
\hline $\begin{array}{l}\text { Plasma uric acid } \\
\text { umols/ml } \mathbf{m}^{2}\end{array}$ & $551 \pm 83 \cdot 8$ & $490 \pm 65 \cdot 0$ & $320 \pm 85 \cdot 2$ \\
\hline $\begin{array}{l}\text { Chondrocalcinosis of } \\
\text { the knees }\end{array}$ & 8 & 1 & 0 \\
\hline
\end{tabular}

'There is no significance between the age range in each group.

The difference between the plasma uric acid in each of the 3 groups is significant $(p<0.001$ by chi-squared test).

'The differences between the frequencies of chondrocalcinosis of the knees in the three groups are: gouty and control groups $p<0.025 ;$ gouty and asymptomatic hyperuricaemic groups $p<0.07$; asymptomatic hyperuricaemic and control groups p NS (chi-squared test).

rheumatoid arthritis by the American Rheumatism Association criteria, ${ }^{9}$ of greater than one year's duration and who at some time had been recorded as having soft tissue swelling of the knees.

Details of the gouty and control groups have been published in our companion paper but are summarised as follows. The gout patients were drawn without selection from the Charing Cross Hospital Gout Clinic. All had been diagnosed as suffering from gout by accepted criteria. The control group were attending Charing Cross Hospital for treatment of malignant skin lesions, traumatic fractures and abrasions, and acute medical emergencies.

The groups with asymptomatic hyperuricaemia and rheumatoid arthritis were carefully age matched with the gouty and control groups. Details are given in Tables 2 and 3.

To detect chondrocalcinosis in the group with asymptomatic hyperuricaemia standard anteroposterior radiographs of the knees were taken by the same technique as for the gouty and control groups. The study of the patients with rheumatoid arthritis was retrospective; the radiographic technique was the same as for the other groups and the patients were drawn without bias.

Plasma uric acid was estimated by an enzymic method.

Table 3 Details of patients with rheumatoid arthritis and the control group

\begin{tabular}{lcc}
\hline & $\begin{array}{l}\text { Rheumatoid } \\
\text { arthritis }\end{array}$ & Control \\
\hline Number & 127 & 142 \\
Male & 25 & 142 \\
Female & 102 & 0 \\
Age $^{*}$ & $55 \cdot 6 \pm 14 \cdot 6$ & $54 \cdot 9 \pm 13 \cdot 4$ \\
Duration of disease (years) & $1-45$ mean $10 \cdot 5$ & \\
Chondrocalcinosis of the knees* & 1 & 0 \\
\hline
\end{tabular}

*There is no significance between either the ages or the frequency of chondrocalcinosis of the knees in the 2 groups.

\section{Results}

Chondrocalcinosis of the knees, which was linear, was detected in only one patient with asymptomatic hyperuricaemia compared with none of the control group, a difference which is not significant, and 8 of the gouty group, a difference which approached significance $(p<0 \cdot 07)$. The patient with asymptomatic hyperuricaemia and chondrocalcinosis of the knees, a 66-year-old man, had spondylolisthesis and no symptoms in the knees.

Of the group with rheumatoid arthritis, chondrocalcinosis of the knees, which was within the meniscal cartilage, was detected in one patient. This is not a statistically significant difference from the control group.

This patient had presented at the age of 64 with recurrent acute monoarthritis of the hands and feet which settled completely between attacks. Radiographs of the knees taken at that time showed chondrocalcinosis. Synovial fluid was not examined for crystals. Serum urate was normal, rheumatoid factor test negative, and the erythrocyte sedimentation rate settled between attacks. One year later persistent symmetrical polyarthritis supervened, associated with a strongly positive test for rheumatoid factor, radiological erosions, and a persistently elevated erythrocyte sedimentation rate. It is impossible to be certain whether this patient's presenting symptoms were of palindromic rheumatoid arthritis or of pseudogout.

\section{Discussion}

As the incidence of chondrocalcinosis increases with age, ${ }^{10}{ }^{11}$ the 4 groups in this study have been carefully age matched to avoid error. Females are overrepresented in the groups with asymptomatic hyperuricaemia and rheumatoid arthritis, but most reports give an approximately equal sex incidence of chondrocalcinosis overall, ${ }^{10}{ }^{12}$ perhaps with a preponderance of females in the older age groups. ${ }^{13}$ The mean age of the control group is lower than that of the other large series, in which a relatively high prevalence of chondrocalcinosis of the knees has been reported, and this probably accounts for the absence of calcification in these subjects. Since only routine radiographs were used, early calcification may have been missed, but this would have also applied to the other groups. The level of serum uric acid is appreciably lower in the group with asymptomatic hyperuricaemia than in the patients with gout; it is not possible to say whether this is a relevant factor.

These results indicate that the frequency of chondrocalcinosis of the knees is increased in patients with hyperuricaemia who have had gout but 
not increased in patients with hyperuricaemia who have not had gout. In chondrocalcinosis an increased incidence of hyperuricaemia has been reported, ${ }^{14}{ }^{15}$ but this was often secondary to diuretic therapy or renal impairment and the association may be spurious. The data presented here imply that idiopathic hyperuricaemia does not predispose to chondrocalcinosis.

If the postulate is correct that urate crystals act as a nucleating agent for the deposition of pyrophosphate, then those patients with gout and chondrocalcinosis might have been expected to have suffered gout of the knees at some time. Although 6 of these 8 patients had had episodes of synovitis of the knees, in only one had urate crystals been identified in synovial fluid. Moreover, 6 of these patients had chondrocalcinosis in other joints where there was no question of a history of gout. However, deposition of urate in asymptomatic joints is well described, ${ }^{16}$ and the absence of symptoms does not preclude the presence of both urate and pyrophosphate crystals. Alternatively, these patients may have a tendency to crystallise both pyrophosphate and urate within their joints with no direct causal relationship between the 2 .

The frequency of chondrocalinosis was not shown to be increased in rheumatoid arthritis, a finding contrary to those of previous studies. ${ }^{2}{ }^{8}$ Subjects in these previous studies, however, were not controlled with regard to their age, which, as already remarked, is of critical importance in any work carried out on the frequency of chondrocalcinosis. Chondrocalcinosis is certainly occasionally seen in relatively young patients with rheumatoid arthritis, but from the evidence of the present study the association is probably fortuitous. If there is indeed a real association, it must be slight.
We thank the physicians of Northwick Park Hospital who agreed to this study on their patients and gratefully acknowledge the help of the departments of Radiology and Medical Records at Northwick Park hospital.

\section{References}

' Stockman A, Darlington L G, Scott J T. Frequency of chondrocalcinosis of the knees and avascular necrosis of the femoral head in gout: a controlled study. Ann Rheum Dis 1980; 39: 7-11.

${ }^{2}$ Good A E, Rapp R. Chondrocalcinosis of the knee with gout and rheumatoid arthritis. $N$ Engl J Med 1967; 277: 286-90.

${ }^{3}$ Dodds W J, Steinbach H L. Gout associated with calcification of articular cartilage. $N$ Engl J Med 1966; 275: 745-9.

4 Grahame R, Mitchiner M B, Sutor J. Crystal deposition in hyperparathyroidism. Ann Rheum Dis 1971; 30: 597-604.

5 Reginato A J, Schumacher H R, Martinez V A. The articular cartilage in familial chondrocalcinosis. Light and electron microscopic study. Arthritis Rheum 1974; 17: 977-92.

${ }^{6}$ Bjelle A O. Morphological study of articular cartilage in pyrophosphate arthropathy. Ann Rheum Dis 1972; 31: 449-56.

7 Katz W A. Deposition of urate crystals in gout. Altered connective tissue metabolism. Arthritis Rheum 1975; 18: 751-6.

${ }^{8}$ Moskowitz R W, Garcia F. Chondrocalcinosis articularis (pseudogout syndrome). Arch Intern Med 1973; 132: 87-91.

9 Ropes M W, Bennett G A, Cobbs S, Jacox R, Jessar R A. Diagnostic criteria for rheumatoid arthritis. 1958 revision. Ann Rheum Dis 1959; 18: 49-53.

${ }^{10}$ McCarty D J. Pseudogout articular chondrocalcinosis. In: Hollander J L, McCarty D J eds. Arthritis and Allied Conditions. 8th ed. Philadelphia: Lea and Febiger, 1972: 1140-60.

11 Pritchard M H, Jessop J D. Chondrocalcinosis in primary hyperparathyroidism. Ann Rheum Dis 1977; 36: 146-51.

12 Bocher J, Mankin H J, Berk R N, et al. Prevalence of calcified meniscal cartilage in elderly persons. $N$ Engl J Med 1965; 272: 1093-7.

${ }^{13}$ Dieppe P, Alexander G, Doherty M, Jones H, Scott D. Subsets of pyrophosphate arthropathy. Rheumatologie in press.

14 McCarty D J, Gatter R A, Brill J M. Crystal deposition diseases: gout and pseudogout. $G P$ 1965; 31: 96-106.

${ }_{15}$ Moskowitz R W, Katz D. Chondrocalcinosis and chondrocalsynovitis (pseudogout syndrome). Am J Med 1967; 43: 322-34.

${ }^{16}$ Agudelo C A, Weinberger A, Schumacher H R, Turner R, Molina J. Definitive diagnosis of gout by identification of urate crystals in asymptomatic metatarsophalangeal joints. Arthritis Rheum 1979; 22: 559-60. 